Flavia de Almeida Vieira

Estudos sobre o comportamento do antimoniato de meglumina no corpo humano e em macacos Rhesus

Tese de Doutorado

Tese apresentada como requisito parcial para obtenção do título de Doutor pelo Programa de PósGraduação em Química da PUC-Rio.

Orientador: Prof. Dr. Norbert Miekeley Co-orientador: Dr. Armando de Oliveira Schubach

Rio de Janeiro

Maio de 2008 


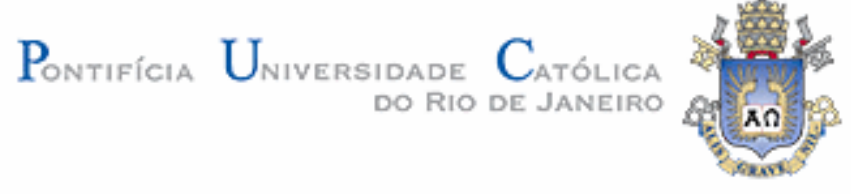

Flavia de Almeida Vieira

\section{Estudos sobre o comportamento do antimoniato de meglumina no corpo humano e em macacos Rhesus}

Tese apresentada como requisito parcial para obtenção do título de Doutor pelo Programa de Pós-Graduação em Química da PUC-Rio. Aprovada pela Comissão Examinadora abaixo assinada.

Prof. Norbert Miekeley

Orientador PUC-Rio

Prof. Armando de Oliveira Schubach

Co-Orientador

Fiocruz

Prof. Francisco José Roma Paumgartten

Fiocruz

Prof. Adilson José Curtius

UFSC

Prof. Jorge Eduardo de Souza Sarkis

CNEN

Prof. Armi Wanderley da Nóbrega

Fiocruz

Prof. Ricardo Erthal Santelli

UFF

Prof. Anderson Schwingel Ribeiro

Sem vínculo

Prof. José Eugenio Leal

Coordenador Setorial do Centro Técnico Científico - PUC-Rio

Rio de Janeiro, 19 de maio de 2008 
Todos os direitos reservados. É proibida a reprodução total ou parcial do trabalho sem autorização da universidade, da autora e do orientador.

\section{Flavia de Almeida Vieira}

Graduou-se em Química Industrial pela Pontifícia Universidade Católica do Rio de Janeiro (PUC-Rio) em 2001. Obteve o título de Mestre pelo Programa de Pós-graduação em Química da PUC-Rio em 2003.

Ficha Catalográfica

Vieira, Flavia de Almeida

Estudos sobre o comportamento do antimoniato de meglumina no corpo humano e em macacos Rhesus / Flavia de Almeida Vieira ; orientador: Norbert Miekeley; co-orientador: Armando de Oliveira Schubach. -2008.

193 f. : il. ; $30 \mathrm{~cm}$

Tese (Doutorado em Química)-Pontifícia Universidade Católica do Rio de Janeiro, Rio de Janeiro, 2008.

Inclui bibliografia

CDD: 540 


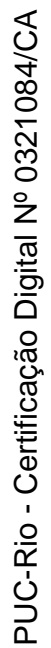

À Zelina Maria, por toda sua vida dedicada a mim. 


\section{Agradecimentos}

Aos meus pais, irmãos e familiares pelo carinho e confiança;

Ao Departamento de Química da PUC-Rio, pelos auxílios concedidos;

Aos meus orientadores, os professores Dr. Norbert Miekeley e Dr. Armando Schubach, pelos ensinamentos e apoio incondicional durante todo trabalho;

À Dra. Karen Friedrich e ao Dr. Francisco Paumgartten do Laboratório de Toxicologia Ambiental da Fiocruz pela estreita parceria no estudo da cinética de antimônio em macacos Rhesus;

À Elaine Marcela Cabral e ao Felipe Maia do Laboratório de ICPMS da PUCRio pela eficiente participação em todas as etapas analíticas da presente tese;

Às Dra. Mariza Salgueiro, Dra. Claudia Maria Valete, Dra. Érica Vasconcelos, Dra. Maria Inês, Dra. Carla e ao Dr. Maurício do Ambulatório de LTA da Fiocruz, pelos ensinamentos sobre leishmaniose; 
À Professora Dra. Carmem Lúcia Porto da Silveira, e aos técnicos Maurício Dupin e André Vechi pelo apoio aos trabalhos desenvolvidos no Laboratório de ICP OES da PUC-Rio;

Ao técnico Álvaro Pereira, pelo constante apoio no Laboratório de ICPMS da PUC-Rio;

À Dra. Christiane Duyck e ao Dr. Anderson Ribeiro do Laboratório de ICPMS da PUC-Rio, pelos ensinamentos sobre ICPMS;

À Adriana dos Santos e aos Denilson Oliveira e Noberto Lemos, pelos serviços prestados e por estarem sempre prontos para ajudar;

À amiga Patrícia Viegas, pela ajuda na formatação da tese;

À Fátima Almeida da Secretaria do Departamento de Química, pela ajuda incondicional no desenlace de todos os entraves burocráticos durante o doutorado;

Aos amigos, colegas, técnicos, funcionários e professores do Departamento de Química da PUC-Rio;

À Nikita e ao Igor, pela constante companhia;

À CAPES, FAPERJ e CNPq pelas bolsas recebidas;

Ao CENPES pelo auxílio financeiro indispensável para os Laboratórios de ICPMS e OES;

À todos os que não foram mencionados nominalmente, mas que contribuíram de alguma forma para o desenvolvimento deste trabalho. 


\section{Resumo}

Vieira, Flavia de Almeida. Estudos sobre o comportamento do antimoniato de meglumina no corpo humano e em macacos Rhesus. Rio de Janeiro, 2008. 193p. Tese de Doutorado - Departamento de Química, Pontifícia Universidade Católica do Rio de Janeiro.

A administração de antimoniais no tratamento da leishmaniose é uma rara oportunidade para estudar o metabolismo de antimônio e das suas espécies químicas no corpo humano. No presente trabalho, a técnica de espectrometria de massa (ICPMS), acoplada ou não à cromatografia iônica (IC) e à geração de hidretos (HG), foi utilizada para determinação das concentrações de antimônio total e de suas espécies químicas, $\mathrm{Sb}(\mathrm{III}), \mathrm{Sb}(\mathrm{V})$ e trimetilantimônio $\left(\mathrm{TMSb}^{\mathrm{V}}\right.$ ) em amostras clínicas de pacientes com leishmaniose e/ou de macacos Rhesus (Mucaca mulatta) tratados com antimoniato de meglumina (AM). Diferentes regimes terapêuticos foram avaliados, incluindo a administração de doses baixa e alta (5 mg ou $20 \mathrm{mg}$ de $\mathrm{Sb}(\mathrm{V})$ por kg de massa corpórea). A concentração total de $\mathrm{Sb}$ foi determinada após a decomposição de amostras por $\mathrm{HNO}_{3} / \mathrm{H}_{2} \mathrm{O}_{2}$. Análises de especiação foram realizadas em amostra de plasma e urina, através do acoplamento em linha da CI ao instrumento de ICPMS. Duas colunas de troca aniônica PRP-X100 foram utilizadas para a separação de espécies empregando o EDTA (eluição isocrática: 4,7 mmol L ${ }^{-1}, 2,5 \%$ v/v metanol, $\mathrm{pH} 4,7$ ) ou tampão de EDTA/fosfato (eluição com variação abrupta de eluente: $1^{\mathrm{o}}$ eluente $-20 \mathrm{mmol} \mathrm{L}{ }^{-1}$ $\mathrm{EDTA}+2 \mathrm{mmol} \mathrm{L}{ }^{-1}, \mathrm{pH} 4,5 ; 2^{\text {o. }}$ eluente $\left.-50 \mathrm{mmol} \mathrm{L}^{-1}\left(\mathrm{NH}_{4}\right)_{2} \mathrm{PO}_{4}, \mathrm{pH} 8,3\right)$ como fase móvel. As características de desempenho de todos os métodos foram avaliadas e serão apresentadas. Testes de estabilidades mostraram que as espécies de antimônio estudadas são suficientemente estáveis permitindo análises de especiação dentro de 24 horas após a coleta. Em humanos, assim como em macacos Rhesus, as concentrações de antimônio em amostras de urina e/ou plasma coletadas após o período de administração de AM, mostraram uma rápida cinética inicial de excreção $\left(t_{1 / 2} \sim 3\right.$ dias $)$ da droga, seguida de duas fases mais lentas. Determinou-se concentrações de Sb significativamente maiores em frações 
de hemácias do que nas amostras de plasma correspondentes, com exceção da fase inicial de administração da droga (primeiras 12 horas). A bioredução de $\mathrm{Sb}(\mathrm{V})$ a $\mathrm{Sb}(\mathrm{III})$ foi observada e confirmada como um importante processo metabólico durante a fase de eliminação. Nenhuma evidência de formação de TMSb (V) foi obtida em nossos estudos. As concentrações de antimônio em amostras de tecidos de macacos Rhesus coletadas aproximadamente 60 dias após a última administração de AM foram maiores na tireóide, seguida por fígado e baço. As concentrações no fígado foram pelo menos 1000 vezes maiores que a concentração basal. Amostras de cabelos/pelos e unhas de pacientes e símios tratados com AM também apresentaram altas concentrações, as quais corresponderam ao histórico de administração da droga e também mostraram que a incorporação de $\mathrm{Sb}$ nestes tecidos é acumulativa, mesmo após o fim da administração de AM. Em cabelos, mesmo após mais de 300 dias do fim do tratamento as concentrações de Sb não retornaram ao nível basal.

\section{Palavras-chave}

antimônio; antimoniato de meglumina, especiação, ICPMS, leishmaniose 


\section{Abstract}

Vieira, Flavia de Almeida. Studies on the behavior of meglumine antimoniate in the human body in Rhesus monkeys. Rio de Janeiro, 2008. 193p. PhD Thesis - Departamento de Química, Pontifícia Universidade Católica do Rio de Janeiro.

Clinical applications of antimonials in the treatment of leishmaniasis are a unique opportunity to investigate the metabolism of antimony and its species in the human body. In this work, inductively coupled plasma mass spectrometry (ICPMS), without or in combination with ion chromatography (IC) and flow injection hydride generation (FI-HG), has been used for the determination of total antimony concentrations [Sb] and three of its expected species, $\mathrm{Sb}$ (III), Sb (V) and trimethyl antimony $\left(\mathrm{TMSb}^{\mathrm{V}}\right)$, in clinical samples of leishmaniasis patients and/or of rhesus monkeys (Mucaca mulatta) treated with meglumine antimoniate (AM). Different drug administration schemes were evaluated, including low and high dose administration ( $5 \mathrm{mg}$ or $20 \mathrm{mg}$ of $\mathrm{Sb}^{5+}$ per $\mathrm{kg}$ of body mass). Total [Sb] was assayed after wet decomposition of the samples by $\mathrm{HNO}_{3} / \mathrm{H}_{2} \mathrm{O}_{2}$. Speciation analysis was performed on plasma and urine samples using IC on-line coupled to the ICPMS instrument. Two PRP-X100 anion exchange columns were used for

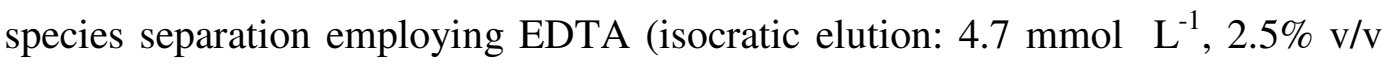
methanol, $\mathrm{pH} 4.7$ ) or EDTA/phosphate buffer (step elution: $1^{\text {st }}$ eluent - $20 \mathrm{mmol}$ $\mathrm{L}^{-1} \mathrm{EDTA}+2 \mathrm{mmol} \mathrm{L}{ }^{-1} \mathrm{KHP}$, at $\mathrm{pH} 4.5 ; 2^{\text {nd }}$ eluent $-50 \mathrm{mmol} \mathrm{L}^{-1}(\mathrm{NH} 4)_{2} \mathrm{HPO}_{4}$ at $\mathrm{pH} \mathrm{8.3)} \mathrm{as} \mathrm{the} \mathrm{mobile} \mathrm{phases.} \mathrm{Performance} \mathrm{characteristics} \mathrm{of} \mathrm{all} \mathrm{methods} \mathrm{were}$ evaluated and are presented. Stability tests showed that all studied antimony species are sufficiently stable to allow speciation analysis within 24 hours after collection. In humans, as well as in rhesus monkeys, the concentrations of antimony in urine and/or plasma samples measured after the administration period of MA, showed rapid excretion kinetics ( $\mathrm{t}_{1 / 2} \sim 3$ days) of the drug followed by two 
slower ones. Significantly higher concentrations of [Sb] were measured in erythrocyte fractions than in corresponding plasma samples, excepting the very initial phase of drug administration (first 12 hours). Bio-reduction of $\mathrm{Sb}^{5+}$ to $\mathrm{Sb}^{3+}$ was observed and confirmed as an important metabolic process during the slow elimination phase. No evidence for the formation of $\mathrm{TMSb}^{\mathrm{V}}$ was so far obtained in our studies. Antimony concentrations in tissue samples of rhesus monkeys $60 \mathrm{~d}$ after the last AM administration were highest in thyroid, followed by liver and spleen. Liver concentrations were at least 1000 times the basal concentrations. Hair and nail samples from treated patients and monkeys had also very high $\mathrm{Sb}$ concentrations, which matched drug administration history and showed also that incorporation of $\mathrm{Sb}$ into these tissues is continuing, even after ceasing $\mathrm{AM}$ application. In human hair, more than 300 days are required for the return of antimony concentrations to basal levels.

\section{Keywords}

Antimony; meglumine antimoniate; speciation; ICPMS; leishmaniasis 


\section{Sumário}

1. Introdução: considerações iniciais, objetivos e justificativas 26 para esse trabalho

2. Leishmanioses 30

2.1. Características gerais das Leishmanioses 30

2.2. Distribuição geográfica e número de casos 34

2.3. Tratamento da Leishmaniose Tegumentar Americana 37

$\begin{array}{ll}\text { 2.3.1. Antimoniais pentavalentes } & 37\end{array}$

2.3.2. Regimes terapêuticos com antimoniais pentavalentes 38

$\begin{array}{ll}\text { 2.3.3. Drogas de segunda escolha } & 41\end{array}$

2.4. Aspectos farmacocinéticos dos antimoniais pentavalentes 42

2.4.1. Conceitos básicos de farmacocinética 42

2.4.2. Farmacocinética do antimônio 45

3. Antimônio e suas espécies químicas $\quad 51$

3.1. Usos, propriedades químicas e toxicidade do antimônio 51

3.2. Antimônio em sistemas ambientais 54

3.3. Antimônio em sistemas biológicos 61

3.4. Monitoramento biológico do antimônio 65

3.4.1. Considerações gerais sobre emprego de amostras de 67 cabelo como biomonitor

3.4.2. Uso de cabelo como biomonitor de antimoniais 69 pentavalentes

4. Técnicas empregadas para análise de especiação de 71 antimônio

4.1. Importância da análise de especiação 71 
4.2. Técnicas analíticas empregadas em análises de especiação de $\mathrm{Sb}$

4.2.1. Métodos cromatográficos 76

4.2.1.1. Aplicações da técnica de cromatografia iônica na análise 77 de especiação de antimônio

4.2.2. Eletroforese Capilar 80

4.2.3. Geração de Hidretos 83

4.2.4. Detectores elementares para análise de especiação 84

4.2.5. Interface entre o ICPMS e o sistema cromatográfico de 87 separação

4.2.6. Análise por diluição isotópica $\quad 89$

5. Materiais e métodos 91

5.1. Instrumentação principal utilizada neste trabalho 91

5.1.1. Espectrometria de massa com plasma indutivamente 91 acoplado

5.1.2. Espectrometria de emissão óptica com plasma 92 indutivamente acoplado

5.1.3. Cromatografia iônica 93

5.2. Soluções e reagentes 94

5.3. Metodologia para determinação de Sb-total em amostras 95 clínicas e biológicas

5.3.1. Determinação de Sb-total em fluidos biológicos 95

5.3.2. Determinação de Sb-total em cabelos e unhas 96

5.3.3. Determinação de Sb-total em tecidos (órgãos de macacos) 97

5.4. Metodologias para determinação das espécies de Sb em 98 amostras clínicas

5.4.1. Metodologias para determinação das espécies de Sb em 98 amostras de plasma e urina

5.4.2. Análise de especiação em antimoniato de meglumina por 99 HG-ICPMS 
5.5. Testes de estabilidade das espécies de antimônio 100

5.6. Grupos de amostragem 101

5.6.1. Estudo terapêutico com macacos Rhesus inoculados com 101

Leishmania (V.) braziliensis

5.6.2. Estudo terapêutico com pacientes com leishmaniose 102 tegumentar americana

$\begin{array}{lr}\text { 6. Resultados e discussões } & 106\end{array}$

6.1. Desenvolvimento de técnicas analíticas para análise de 106 especiação de antimônio por IC-ICPMS

6.1.1 Separação das espécies inorgânicas de Sb com a coluna 106 PRP-X100 (150 mm)

6.1.2. Separação $\mathrm{Sb}(\mathrm{V}), \mathrm{Sb}(\mathrm{III})$ e $\mathrm{TMSb}(\mathrm{V})$ com a coluna 110 PRP-X100 (150 mm)

6.1.3. Separação das três espécies de Sb com a coluna 115 PRP-X100 (250 mm)

6.1.4. Características de desempenho dos dois métodos 118 cromatográficos aperfeiçoados

6.2. Análise de antimoniato de meglumina (Glucantime®) 120

6.2.1. Quantificação das espécies inorgânicas de antimônio 120

6.2.2. Comportamento cromatográfico do antimoniato de 122 meglumina

6.3. Avaliação da estabilidade das espécies de antimônio de 124 interesse biológico

6.4. Características de desempenho do método para 126 determinação de Sb-total

6.4.1. Resultados obtidos para materiais certificados 126

6.4.2. Desempenho do procedimento para digestão ácida de 129 amostras clínicas

6.5. Comportamento de antimônio em macacos rhesus 132 inoculados com Leishmania

6.5.1. Cinética de excreção de antimônio total em plasma e papa 
de hemácias após aplicação de dose única de antimoniato de meglumina no primeiro dia

6.5.2. Cinética de excreção de antimônio total em plasma, papa de hemácias e pelos após aplicação de doses repetidas de antimoniato de meglumina

6.5.3. Cinética de excreção de antimônio em pelos 143

6.5.4. Acumulação de antimônio em órgãos de macacos Rhesus 145

6.5.5. Produtos de transformação de antimoniato de meglumina 149

6.6. Estudo terapêutico com pacientes com Leishmaniose 152 Tegumentar Americana

6.6.1. Cinética de excreção de antimônio em fluidos biológicos 152

6.6.2. Biomonitoramento de antimônio através de cabelos e 163 unhas

6.6.3. Especiação de antimônio em urina de paciente com LTA 169 tratado com antimoniato de meglumina

7. Conclusões

8. Referências bibliográficas 


\section{Lista de Tabelas}

Tabela 1: Exemplos de agentes causadores de leishmaniose (adaptado de Herwaldt, 1999).

Tabela 2: Número de casos de LTA por região brasileira (MS, 2006).

Tabela 3: Fórmulas estruturais químicas de compostos antimoniais.

Tabela 4: Fórmulas estruturais químicas de drogas de segunda escolha utilizadas no tratamento de leishmaniose (fonte: Croft et al., 2003).

Tabela 5: Parâmetros farmacocinéticos do antimônio.

Tabela 6: Propriedades químicas do antimônio (Sb). 52

Tabela 7: Concentração de antimônio no meio ambiente e em organismos da biota.

Tabela 8: Principais espécies de Sb em matrizes ambientais.

Tabela 9: Concentração de antimônio em amostras clínicas de humanos.

Tabela 10: Valores médios para concentração de antimônio em matrizes biológicas.

Tabela 11: Concentração de antimônio em cabelos de pacientes de leishmaniose tratados com duas diferentes dosagens de antimônio (Dorea et al, 1989).

Tabela 12: Metodologias de análise de especiação de antimônio.

Tabela 13: Alguns termos utilizados em métodos 77 cromatográficos.

Tabela 14: Metodologias utilizadas na análise de especiação de antimônio por cromatografia aniônica.

Tabela 15: Equipamentos e parâmetros operacionais utilizados em ICPMS. 
Tabela 16: Parâmetros operacionais utilizados em ICP-OES.

Tabela 17: Metodologia de digestão ácida para amostra de fluidos biológicos.

Tabela 18: Metodologia de lavagem e digestão de cabelos e unhas.

Tabela 19: Procedimento para digestão de tecidos.

Tabela 20: Metodologia de análise de especiação de Sb por IC-ICPMS (método A).

Tabela 21: Metodologia para análise de especiação de Sb por IC-ICPMS (método B)

Tabela 22: Metodologia para determinação de Sb(III) por HGICPMS em Glucantime®.

Tabela 23: Intervalos de coleta e análises.

Tabela 24: Efeito da concentração do EDTA no tempo de 108 retenção ajustado (tr') das espécies de antimônio.

Tabela 25: Efeito da adição de metanol no tempo de retenção 109 (tr') ajustado, fator de retenção $(k)$ e largura da meia altura $\left(w^{1 / 2}\right)$ dos picos das espécies de antimônio em EDTA $4 \mathrm{mmol} \mathrm{L}^{-1}$.

Tabela 26: Fator de retenção $(k)$ das espécies de antimônio nos diferentes programas de eluição.

Tabela 27: Características analíticas de desempenho da 119 separação cromatográfica de duas espécies de antimônio pelo método $A$.

Tabela 28: Características analíticas de desempenho da 120 separação cromatográfica de três espécies de antimônio pelo método $B$.

Tabela 29: Concentração (em mg mL ${ }^{-1}$ ) de $\mathrm{Sb}$ (III) e Sb-total em 122 antimoniato de meglumina (Glucantime ${ }^{\circledR}$, Aventis Farma Ltda., São Paulo, Brasil)

Tabela 30: Parâmetros de desempenho da metodologia para 131 determinação de $\mathrm{Sb}$ e $\mathrm{Pb}$ em amostras de referência. 
Concentrações em $\mu \mathrm{g} \mathrm{L}^{-1}$ ou $\mathrm{mg} \mathrm{L}^{-1}$ quando indicado $\left(^{*}\right)$.

Tabela 31: Média das concentrações de antimônio (em $\mathrm{mg} \mathrm{L}^{-1}$ ) e $T_{\max }$ alcançadas em fluidos durante o ensaio com dose única de AM.

Tabela 32: Parâmetros cinéticos obtidos para aplicação de dose 138 única de AM.

Tabela 33: Variação das concentrações médias de $\mathrm{Sb}\left(\mu \mathrm{g} \mathrm{L}^{-1}\right)$ 140 em amostras de fluidos biológicos de macacos Rhesus tratados com antimoniato de meglumina (AM).

Tabela 34: Variação da concentração média de [Sb] em 145 amostras de pelos de macacos Rhesus coletadas antes, durante e depois do tratamento com $A M(n=6$ para cada grupo; $p=0,80)$.

Tabela 35: Concentração média e limites de confiança $(p=0,80)$ 147 de antimônio em tecidos liofilizados de macacos Rhesus $(n=3)$ coletados aproximadamente 60 dias depois do último tratamento com antimoniato de meglumina. Análises em triplicata; (a) dado referente a um único animal, todas as concentrações em $\mu \mathrm{g} \mathrm{g}^{-1}$.

Tabela 36: Intervalo de tempo necessário para retorno da 153 concentração de $\mathrm{Sb}$ ao nível basal em fluidos biológicos de pacientes com LTA após o tratamento com $\operatorname{AM}(p=0,80)$.

Tabela 37: Concentração média (máxima) de antimônio 155 alcançada em sangue total e plasma de pacientes com LTA durante $\mathrm{o}$ tratamento com antimoniato de meglumina; (a) limite de confiança calculado para $p=0,80$.

Tabela 38: Concentração média (máxima) de antimônio 155 alcançada em urina de pacientes com LTA durante o tratamento com antimoniato de meglumina; (a) limite de confiança calculado para $p=0,80$.

Tabela 39: Número de participantes do ensaio de 163 biomonitoramento de cabelos e unhas por grupo de amostragem

Tabela 40: Concentração média (máxima) de antimônio no 165 cabelo $\left(\mu \mathrm{g} \mathrm{g}^{-1}\right)$ de pacientes com LTA após tratamento com 
antimoniato de meglumina (intervalo de confiança para $p=0,80$ ).

Tabela 41: Concentração média (máxima) de antimônio $\left(\mu \mathrm{g} \mathrm{g}^{-1}\right.$ ) 166 em unhas de pacientes com LTA após tratamento com antimoniato de meglumina (intervalo de confiança para $p=0,80$ ).

Tabela 42: Intervalo de tempo necessário para retorno da 168 concentração de $\mathrm{Sb}$ ao nível basal em cabelos e unhas de pacientes com LTA após o tratamento com AM. Intervalo de confiança calculado para $p=0,80$. 


\section{Lista de Figuras}

Figura 1: Fêmea do flébotomo transmissor da leishmaniose (MS, 2007).

Figura 2: a) Lesão típica de leishmaniose cutânea; b) Forma cutânea difusa; c) Forma mucosa tardia (Fonte: MS, 2007).

Figura 3: Classificação clínica e os respectivos agentes etiológicos da LTA no Brasil (MS, 2006).

Figura 4: Mapa de distribuição de $90 \%$ dos casos de Leishmaniose (OMS, 2002).

Figura 5: Casos notificados de LTA no Brasil entre 1980 e 2005 (fonte: MS, 2007).

Figura 6: Cromatogramas de duas espécies de $\mathrm{Sb}$ em soluções 107 aquosas. Os sinais correspondem a $2 \mathrm{ng}$ de antimônio para cada espécie injetada na coluna PRP-X100, Hamilton. a) EDTA $1 \mathrm{mmol} \mathrm{L}{ }^{-1}$; b) EDTA $2,5 \mathrm{mmol} \mathrm{L}^{-1}$; c) EDTA $4 \mathrm{mmol} \mathrm{L}^{-1}$; d) EDTA $10 \mathrm{mmol} \mathrm{L}^{-1}$.

Figura 7: Tempo de retenção ajustado versus concentração de 108 EDTA.

Figura 8: Fator de retenção versus concentração de EDTA. 109

Figura 9: Cromatograma das espécies de $\mathrm{Sb}$ (V) e $\mathrm{Sb}$ (III) em 110 soluções aquosas. Os sinais correspondem a $3 \mathrm{ng}$ de antimônio para cada espécie injetada na coluna PRP-X100, Hamilton, com o eluente EDTA 4 mmol L ${ }^{-1}$ com metanol 2,5\% v/v (pH 4,7).

Figura 10: Cromatogramas de três espécies de $\mathrm{Sb}$ em soluções aquosas. Os sinais correspondem a $1 \mathrm{ng}$ de antimônio para cada das três espécies injetadas. Sample read delay 30s. EDTA $\mathrm{pH}$ 4,7 empregado como fase móvel, nas seguintes concentrações: a) $1 \mathrm{mmol} \mathrm{L}^{-1}$; b) $4 \mathrm{mmol} \mathrm{L}^{-1}$; c) $6 \mathrm{mmol} \mathrm{L}^{-1}$; d) $10 \mathrm{mmol} \mathrm{L}^{-1}$; e) $18 \mathrm{mmol} \mathrm{L}^{-1}$; f) $24 \mathrm{mmol} \mathrm{L}^{-1}$. 
Figura 11: Cromatogramas das espécies de $\mathrm{Sb}$ em soluções aquosas empregando-se o eluente ácido cianúrico $3 \mathrm{mmol} \mathrm{L}^{-1}$ (pH 4,7). Sample read delay: 30s. Espécies injetadas: a) Cs, $\mathrm{TMSb}$; b) $\mathrm{Cs}, \mathrm{Sb}(\mathrm{V}) \mathrm{c}$ ) $\mathrm{Cs}, \mathrm{Sb}(\mathrm{III})$; d) não foi injetada nenhuma espécie, as espécies retidas de $\mathrm{Sb}$ foram eluídas com EDTA 4 $\mathrm{mmoL} \mathrm{L}^{-1}, \mathrm{pH} 4,7$. A espécie catiônica $\mathrm{Cs}^{+}$foi utilizada como marcador do tempo morto.

Figura 12: Cromatogramas das espécies de $\mathrm{Sb}$ em soluções 113 aquosas, empregando-se 0 eluente ácido cianúrico $3 \mathrm{mmol} \mathrm{L}^{-1}$ (pH 7,0). Sample read delay: 30s. Espécies injetadas: a) Cs, $\mathrm{TMSb}$; b) $\mathrm{Cs}, \mathrm{Sb}(\mathrm{V}) \mathrm{c}) \mathrm{Cs}, \mathrm{Sb}(\mathrm{III})$; d) não foi injetada nenhuma espécie, a espécie retida (SbIII) foi eluída com EDTA $4 \mathrm{mmoL} \mathrm{L}^{-}$ ${ }^{1}, \mathrm{pH} 4,7$. A espécie catiônica $\mathrm{Cs}^{+}$foi utilizada como marcador do tempo morto.

Figura 13: Cromatogramas das espécies de $\mathrm{Sb}$ em soluções 114 aquosas, empregando-se o eluente ácido cianúrico $3 \mathrm{mmol} \mathrm{L}^{-1}$ (pH 10,0). Sample read delay: 30s. Espécies injetadas: a) Cs, TMSb; b) Cs, Sb(V) c) Cs, Sb(III); d) Cs, TMSb, Sb(III) e Sb(V). A espécie catiônica $\mathrm{Cs}^{+}$foi utilizada como marcador do tempo morto.

Figura 14: Cromatogramas das espécies de $\mathrm{Sb}$ em soluções aquosas utilizando-se o eluente ácido cianúrico $3 \mathrm{mmol} \mathrm{L}^{-1}$, perclorato $2,5 \mathrm{mmol} \mathrm{L}^{-1}$, acetonitrila $2 \%(\mathrm{pH} \mathrm{9,0)}$. Sample read delay: 30s. Espécies injetadas: a) Cs, TMSb; b) Cs, $\mathrm{Sb}(\mathrm{V}) \mathrm{c}) \mathrm{Cs}$, $\mathrm{Sb}$ (III); d) Cs, TMSb, $\mathrm{Sb}$ (III) e Sb(V). A espécie catiônica $\mathrm{Cs}^{+}$foi utilizada como marcador do tempo morto.

Figura 15: Cromatogramas típicos obtidos nas condições 118 otimizadas do método B. a) urina fortificada com $3 \mathrm{ng}$ de antimônio para cada espécie injetada na coluna; b) antimoniato de meglumina (fator de diluição $=10^{7}$ ); sample read delay $55 \mathrm{~s}$.

Figura 16: Cromatogramas da droga diluída em solução aquosa 123 (fase móvel: EDTA 4 mmol L-1 $+\mathrm{MeOH} 2,5 \% \mathrm{v} / \mathrm{v}, \mathrm{pH} 4,7$ ): a) droga diluída (fator $10^{7}$ ); b) droga diluída (fator $10^{6}$ ) em solução 
aquosa, fortificada com $20 \mu \mathrm{g} / \mathrm{L}$ de $\mathrm{Sb}(\mathrm{V})$; c) droga diluída (fator $10^{6}$ ) em solução aquosa, fortificada com $20 \mu \mathrm{g} / \mathrm{L}$ de cada espécie de $\mathrm{Sb}$

Figura 17: Cromatograma das espécies $\mathrm{Sb}(\mathrm{III})$ e $\mathrm{Sb}(\mathrm{V})$ em urina.

Os sinais correspondem a $1 \mu \mathrm{g} \mathrm{L}^{-1}$ de Cs, e $20 \mu \mathrm{g} \mathrm{L}^{-1}$ de cada espécie inorgânica de antimônio. Condições operacionais: coluna PRP-X100 (Hamilton, EUA); fase móvel de EDTA 4 mmol $\mathrm{L}^{-1}+\mathrm{MeOH} 2,5 \% \mathrm{v} / \mathrm{v}, \mathrm{pH} 4,7$; vazão $1,5 \mathrm{~mL} \mathrm{~min}^{-1}$; volume de injeção $100 \mu \mathrm{L}$; ELAN 5000 (PerkinElmer-Sciex, EUA); Potência $1300 \mathrm{~W}$; sample read delay $35 \mathrm{~s}$.

Figura 18: Variação das áreas normalizadas de espécies 125 inorgânicas de antimônio em função do tempo decorrido entre o preparo das soluções (urina fortificada com as espécies inorgânicas de antimônio) e da sua medida.

Figura 19: Curva de absorção/eliminação de antimônio em 134 plasma ou hemácias após aplicação de dose única de AM em macacos Rhesus tratados com dose baixa $(a, c)$ e com dose alta de AM b $(b, d)$; plasma $(a, b)$; hemácias $(c, d)$.

Figura 20: Gráfico de concentração de Sb em plasma versus 137 concentração de $\mathrm{Sb}$ em papa de hemácias no ensaio de aplicação única de AM em macacos rhesus tratados com: a) dose baixa $(n=24)$ e b) dose alta $(n=22)$.

Figura 21: Gráfico In [Sb] em plasma versus tempo em macacos 137 Rhesus tratados com a) dose baixa de AM e b) dose alta de AM. Figura 22: Variações na concentração de Sb em amostras de plasma e hemácias com o tempo: a) em amostras de plasma coletadas de macacos distintos tratados por dose baixa e dose alta de AM; b) em amostras de papa de hemácias coletadas de macacos distintos tratados por dose baixa e dose alta de AM; c) em amostras de plasma e papa de hemácias coletadas de um mesmo macaco tratado por dose baixa de AM; d) em amostras de plasma e papa de hemácias coletadas de um mesmo macaco 
tratado por dose alta de AM; e) em amostras de plasma e papa de hemácias do grupo de macacos tratado por dose baixa de AM; f) em amostras de plasma e papa de hemácias do grupo de macacos tratado por dose alta de AM.

Figura 23: Gráfico de concentração de Sb em plasma versus 141 concentração de $\mathrm{Sb}$ em papa de hemácias de amostras coletadas durante e após o tratamento com AM com regimes terapêuticos diferentes: a) de dose baixa $(n=123)$ e b) de dose alta $(n=126)$.

Figura 24: Cinética de eliminação de antimônio: a) fases de 143 eliminação em regime terapêutico com dose baixa de AM; b) fases de eliminação em regime terapêutico com dose alta de AM; c) gráfico de In [Sb] em plasma versus tempo em regime terapêutico com dose alta da $2^{\mathrm{a}}$ e $3^{\mathrm{a}}$ fases de eliminação.

Figura 25: Variação da concentração média de Sb em pelos de 144 macacos Rhesus $(n=6)$ coletadas antes, durante e após o tratamento com baixa e alta dose de AM. a) escala logarítmica e b) escala decimal.

Figura 26: Gráfico de concentração média de Sb em tecidos de 149 macacos Rhesus tratados com antimoniato de meglumina por regimes terapêuticos de dose baixa e dose alta. As concentrações correspondem aos níveis determinados 60 dias após da ultima aplicação da droga.

Figura 27: Cromatogramas mostrando diferentes espécies de 151 $\mathrm{Sb}$ em plasma de macacos tratados por dose alta $\left(\mathrm{Sb}^{\mathrm{V}}: 20 \mathrm{mg}\right.$ $\mathrm{kg}^{-1} \mathrm{~d}^{-1}$ ) de AM. As amostras foram coletadas após a) 2 ; b) 3; c) 6 e d) 10 dias do fim do tratamento.

Figura 28: Cromatogramas mostrando diferentes espécies de 151 $\mathrm{Sb}$ em plasma de macacos tratados por dose baixa $\left(\mathrm{Sb}^{\mathrm{V}}: 5 \mathrm{mg}\right.$ $\mathrm{kg}^{-1} \mathrm{~d}^{-1}$ ) de antimoniato de meglumina. As amostras foram coletadas após a) 2 ; b) 3; c) 6 e d) 10 dias do fim do tratamento.

Figura 29: Curva de eliminação de $\mathrm{Sb}$ antes, durante e depois 
do tratamento com AM de integrante do grupo de dose alta contínuo. Paciente com LTA; feminino; 26 anos; 64 kg; tratamento contínuo com 20 doses cada uma de $1215 \mathrm{mg} \mathrm{Sb}(\mathrm{V})$ ( dia. a) em urina; b) em urina com escala logarítmica; c) em sangue total e plasma; d) correlação das concentrações de $\mathrm{Sb}$ no plasma versus sangue total.

Figura 30: Curva de eliminação de Sb antes, durante e depois 159 do tratamento com AM de integrante do grupo de dose alta em série. Paciente com LTA; masculino; 57 anos; 70 kg; tratamento em 2 séries com 12 e 8 doses, respectivamente, cada uma de $1215 \mathrm{mg}$ de $\mathrm{Sb}(\mathrm{V})$ / dia com intervalo entre as séries de 31 dias. a) em urina; b) em urina com escala logarítmica; c) em sangue total e plasma; d) correlação das concentrações de Sb no plasma versus sangue total.

Figura 31: Curva de eliminação de $\mathrm{Sb}$ antes, durante e depois 160 do tratamento com AM de integrante do grupo de dose baixa contínuo convencional. Paciente com LTA; feminino; 19 anos; 53 kg; tratamento contínuo com 25 doses cada uma de 267,3 mg $\mathrm{Sb}$ (V) / dia. a) em urina; b) em urina com escala logarítmica; c) em sangue total e plasma; d) correlação das concentrações de $\mathrm{Sb}$ no plasma versus sangue total.

Figura 32: Curva de eliminação de Sb antes, durante e depois do tratamento com AM de integrante do grupo de dose baixa em série. Paciente com LTA; masculino; 63 anos; $63,5 \mathrm{~kg}$; tratamento em 3 séries com 10 doses cada uma de $324 \mathrm{mg} \mathrm{Sb}$ (V) / dia, com intervalos entre as séries de 10 e 13 dias, respectivamente. a) em urina; b) em urina com escala logarítmica; c) em sangue total e plasma; d) correlação das concentrações de Sb no plasma versus sangue total.

Figura 33: Curva de eliminação de $\mathrm{Sb}$ antes, durante e depois do tratamento com AM de integrante do grupo de dose baixa contínuo de curta duração. Paciente com LTA; masculino; 16 anos; $66,8 \mathrm{~kg}$; tratamento contínuo com 10 doses cada uma de 
324 mg Sb (V) / dia. a) em urina; b) em urina com escala logarítmica; c) em sangue total e plasma; d) correlação das concentrações de Sb no plasma versus sangue total.

Figura 34: Variação da concentração de Sb em cabelos e unhas de pacientes com LTA antes, durante e após o tratamento com dose alta de AM. a) Paciente do grupo de dose alta contínuo; masculino; 23 anos; 64,3 kg; tratamento contínuo com 20 doses cada uma de 1215 mg Sb (V) / dia. b) Paciente do grupo de dose alta em série; feminino; 44 anos; 58 kg; tratamento em 2 séries com 8 e 12 doses, respectivamente, cada uma de 1134 mg de $\mathrm{Sb}(\mathrm{V})$ / dia com intervalo de 24 dias.

Figura 35: Variação da concentração de Sb em cabelos e unhas 168 de pacientes com LTA antes, durante e após o tratamento com dose baixa de AM. a) Paciente do grupo de dose baixa contínuo; feminino; 22 anos; 49 kg; tratamento contínuo com 30 doses cada uma de 259,2 mg Sb (V) / dia. b) Paciente do grupo de dose baixa em série; masculino; 63 anos; $63,5 \mathrm{~kg}$; tratamento em 3 séries com 10 doses cada uma de 324 mg Sb (V) / dia, com intervalos entre as séries de 10 e 13 dias, respectivamente. c) Paciente do grupo de dose baixa contínuo de curta duração; masculino; 25 anos; 63,2 kg; tratamento com 10 doses cada uma de 324 mg de Sb (V) / dia.

Figura 36: Cromatogramas de amostras de urina coletadas após a conclusão do tratamento de AM (sample read delay igual a 55s). a) 20 horas após a última aplicação de AM (fator de diluição $=100 x$ ) ; b) 55,6 horas após a última aplicação de AM (fator de diluição $=10 x$ ). Paciente com LTA do grupo de dose baixa em série; masculino; 63 anos; $63,5 \mathrm{~kg}$; tratamento em 3 séries com 10 doses cada uma de $324 \mathrm{mg} \mathrm{Sb}(\mathrm{V})$ / dia, com intervalos entre as séries de 10 e 13 dias, respectivamente.

Figura 37: Variação da razão entre Sb (III) e Sb-total em urina de paciente com LTA após a conclusão do tratamento com AM. 


\section{Lista de Abreviaturas e Símbolos}

AAS - espectrometria de absorção atômica

AM - antimoniato de meglumina

CE - eletroforese capilar

CLAE (ou HPLC) - cromatografia líquida de alta eficiência

CMP - concentração máxima permissível

DIN - nebulização com injeção direta

EOF - vazão eletroosmótica

FIA - análise por injeção em fluxo

$H G$ - geração de hidretos

HEN - nebulizador de alta eficiência

IC (ou Cl)- cromatografia iônica

ICPMS - espectrometria de massa com plasma indutivamente acoplado

ICP-OES - espectrometria de emissão óptica com plasma indutivamente acoplado

ID -diluição isotópica

IUPAC - União Internacional de Química Pura e Aplicada

$\mathrm{k}$ - fator de retenção

LC - leishmaniose cutânea

LD - limite de detecção

LM - leishmaniose mucosa

LTA - Leishmaniose Tergumentar Americana

LV - leishmaniose visceral

MRC - material de referência certificado

MS - Ministério da Saúde

OMS - Organização Mundial de Saúde

$\mathrm{t}_{1 / 2}=$ tempo de meia vida

$T_{\max }=$ tempo para alcançar a concentração máxima

tr' $=$ tempo de retenção ajustado

$\mathrm{t}_{0}=$ tempo morto

UV-vis - ultravioleta e visível 